\title{
L'ÉTUDE DE LA NARRATIVITÉ DANS LES ARTICLES BOURSIERS
}

\author{
Anne-Sophie Collard et Lysiane Romain ${ }^{1}$
}

Le ratissage de la narrativité des articles de presse économique et boursière comprend une série de concepts et d'outils développés en narratologie pour étudier le personnage et la construction du récit. Etape par étape, il nous permet d'établir le degré de narrativité d'un texte, c'est-à-dire l'orientation de la construction d'un texte vers le discours narratif. A la première lecture, et ce de manière générale, la tendance des articles boursiers étudiés semble se diriger plutôt vers la description, l'énumération de faits et d'éléments du discours, que vers la narration. Nous supposons en outre que le degré de narrativité est d'autant plus faible que la presse est spécialisée dans le domaine économique et boursier. Plusieurs éléments viennent étayer ces hypothèses. Nous pensons également que la narrativité joue un rôle dans la compréhension des textes économiques et boursiers.

La recherche que nous avons réalisée s'est déroulée en mars 2002. Elle porte sur l'étude de huit articles ${ }^{2}$ issus de la presse d'information

1 Anne-Sophie Collard et Lysiane Romain sont assistante et chercheuse au Département de communication de l'Université catholique de Louvain.

2 Cinq articles ont été publiés dans Le Vif/L'Express : «Les petites valeurs en folie »

Recherches en communication, $\mathrm{n}^{\circ} 23$ (2005). 
générale et huit ${ }^{1}$ articles de la presse spécialisée. Une des composantes centrales d'un récit étant le personnage, nous mettrons en évidence les différentes facettes, l' « être », le «dire » et le «faire», des acteurs présents dans les articles de notre corpus. Après avoir étudié les relations qu'entretiennent ces personnages, nous nous pencherons sur la construction de la structure du récit.

Pour cerner les différents acteurs, nous avons identifié leur étiquette ${ }^{2}$, c'est-à-dire l'ensemble des attributs du personnage qui font de lui un sujet unique et clairement identifiable. Un des éléments pour l'élaboration de l'étiquette est le nom du personnage, ou plus précisément les désignateurs ${ }^{3}$. Ils déterminent à eux seuls un certain mode de lecture et ils permettent de repérer le nombre d'acteurs présents. Le nom, comme les attributs, font partie de l'ensemble des descriptions de l'acteurs, l'«être ». Les attributs assurent à l'acteur une individualité, une personnalité, qui permettra de l'identifier et de le situer, en tant qu'acteur principal ou secondaire, en tant que personnage caractéristique, en tant que conducteur d'affects, etc.

Dans le corpus d'articles étudié, on remarque qu'aucun texte ne met en scène un seul personnage central. En effet, au-delà de la présence d'acteurs périphériques venant alimenter le récit, il y a toujours au moins deux acteurs centraux. Dans la plupart des cas (11 articles sur 16), on en dénombre même davantage (jusque 5 acteurs centraux) ${ }^{4}$.

(26/03/99), «Une stratégie payante» (23/04/99), «Le Nasdaq contre le Down Jones » (03/03/00), «Intéressants, les cliquets ? (25/08/00), « Acheter au son du canon ? (26/01/01). Trois articles proviennent de La Libre Belgique : « Manque de visibilité pénalisé » (10-11/03/01), «Les warrants ref(er)ont surface»(16/05/01), « Euronext vaut 120 milliards » (22/06/01).

1 Trois articles sont issus de La Libre Entreprise : «Brokers online en panne» (10/03/01), « Nasdaq : un jeudi noir tienté de vert» (24/03/01), «Petit parfum spéculatif en ce début d'été » (30/06/01). Trois ont été publiés dans L'Echo : «Une année de perdue pour les fonds de pension : le return moyen à $-0,1 \%$ en 2000 » (29/03/01), «La présence d'Interbrew chamboule les statistiques d'Euronext Bruxelles » (30/05/01), «Belle croissance bénéficiaire en perspective pour les distributeurs alimentaires européens » (07/06/01). Deux textes boursiers proviennent de Trends Tendances: «La révolte des actionnaires » (24/05/01), «Les Bourses entrent en Bourse » (07/06/01).

2 HAMON Philippe, «Pour un statut sémiologique du personnage », in Poétique du récit, Paris, Seuil (collection Points Essais), 1977, pp. 115-180

3 REUTER Yves, "Le personnage », in La question du personnage, Cahiers de Recherche en Didactique du français, ${ }^{\circ} 1$, Clermont-Ferrand, octobre 1927, p. 26

4 Exemple dans l'article «Une année de perdue pour les fonds de pension : le return moyen s'élève à $-0,1 \%$ en 2000 » (L'Echo), on dénombre quatre acteurs centraux : 
Nous pensons que cette multiplication des acteurs centraux dans un article de presse, à l'opposé d'un long roman, ne permet pas à l'auteur de bien construire chacun d'entre eux, de les personnaliser. En effet, on constate que les descriptions données des acteurs portent davantage sur leur secteur d'activité ${ }^{1}$ ou en donnent une définition ${ }^{2}$. Mais ces caractéristiques ne dépassent jamais le secteur boursier. Les acteurs ne sont pas dotés de personnalité spécifique, et ne véhiculent ainsi que très peu d'émotion. Leur «être » est donc en général peu élaboré dans le type d'articles étudié.

Deux autres axes traversent également le personnage : le «faire» et le « dire ». Au-delà de son « être », l' acteur est présent par ses paroles. Nous en avons observé de deux ordres : les paroles que nous qualifions de « réelles », c'est-à-dire retranscrites telles quelles et entre guillemets, et les paroles rapportées par l'auteur. Des différences apparaissent dans le corpus de textes selon que ceux-ci proviennent de la presse économique spécialisée ou non spécialisée. Dans les articles de la presse non spécialisée les acteurs ont très peu la parole, qu'elle soit réelle ou rapportée (seuls 2 articles sur 8 relatent des paroles d'acteurs). Tandis que tous les articles de la presse spécialisée reprennent des paroles d'acteurs, et 7/8 d'entre eux avec une majorité de paroles réelles ${ }^{3}$. Cette forte présence de paroles appuie le caractère descriptif plutôt que narratif de ces textes. Leur rôle semble en effet d'étayer l'énoncé par un fait, ce sont d'ailleurs principalement des experts qui ont la parole.

L'acteur s'affirme en outre par des actions, son «faire » : il agit dans un endroit défini et à un moment déterminé. L'unité d'action, l'unité de lieu et l'unité de temps forment les trois unités d'un récit classique à travers lesquelles les personnages évoluent.

En observant plus attentivement les actions menées par les acteurs centraux des textes, deux catégories se dégagent de l'analyse: les actions linéaires et les actions qui se répètent dans différents lieux.

les fonds de pension, les actions, les obligations et la firme Mercer.

1 Exemple dans l'article «Les petites valeurs en folie » (Le Vif/L'Express). L'auteur décrit la société Netvision somme «spécialisé dans la sécurisation des opérations électroniques sur Internet ».

2 Exemple dans l'article «Les Bourses entrent en Bourse» (Trends Tendance), l'auteur donne la définition d'Euronext : "l'association des Bourses de Bruxelles, d'Amsterdam et de Paris ».

3 Exemple dans l'article «Belle croissance bénéficiaire en perspective pour les distributeurs alimentaires en Europe » (L'Echo). On y retrouve neuf paroles réelles et trois paroles rapportées. 
Certains textes (4/8 dans la presse spécialisée, et 5/8 dans la presse non spécialisée) mettent en scène des acteurs effectuant des actions différentes mais qui s'enchaînent dans le temps ${ }^{1}$; d'autres textes présentent un type d'action, et le déclinent dans divers lieux (4/8 dans la presse spécialisée et 3/8 dans la presse non spécialisée) $)^{2}$. Dans un cas comme dans l'autre, l'unité d'action est privilégiée.

L'acteur agit dans des lieux et dans une certaine temporalité. Dans 12 articles sur 16, on retrouve une unité de lieu, c'est-à-dire que la ou les action(s) décrites se déroulent dans un même lieu. Souvent, ces lieux sont des places géographiques (Bruxelles, les Etats-Unis, Paris, etc) ${ }^{3}$, mais il peut parfois s'agir d'endroits plus spécifiques (la Bourse, l'entreprise, etc. $)^{4}$. Soulignons néanmoins que dans ces articles où un lieu principal est indiqué, l'auteur cite en général au moins une fois un autre lieu et les compare. Concernant le temps, seul 1 article sur 16 construit son récit selon une linéarité temporelle (passé, présent et futur $)^{5}$. De plus, il s'agit d'un article provenant de la presse non spécialisée. Les autres textes oscillent sans cesse entre le passé, le présent et le futur sans lien chronologique. Contrairement aux actions et aux lieux, les récits ne présentent pas, pour la plupart, d'unité de temps.

Les acteurs sont également étudiés à travers les relations qu'ils entretiennent entre eux. Les relations d'opposition « Sujet $-><$ - Antisujet» font notamment partie des «bonnes recettes » pour la mise en récit. C'est ainsi que les contes se construisent souvent autour d'une relation d'opposition forte entre un héros et un agresseur. Comme, par exemple, dans «Blanche-Neige et les sept nains » où l'héroïne se voit pourchassée par sa belle-mère, la reine voulant devenir à son tour la plus belle du royaume. Nous avons relevé 2 articles sur 16 construits autour d'une relation d'opposition centrale ${ }^{6}$. Ces textes font tous les

1 Exemple dans le texte « Acheter au son du canon? » (Le Vif/L'Expresse).

2 Exemple dans le texte «Les Bourses entrent en Bourse» (Trends Tendance). On y décrit l'action d'entrer en Bourse dans différents pays (Norvège, Angleterre, Allemagne, Belgique, Etats-Unis, etc.).

3 Exemple dans l'article «La révolte des actionnaires» (Trends Tendance) : les actions principales se déroulent dans plusieurs pays : France, Allemagne, Italie, etc.

4 Exemple dans l'article «Les warrants ref(er)ont surface » (La Libre Belgique) : les actions principales se déroulent à la Bourse de Bruxelles.

5 Exemple : l'article «Une stratégie payante»(Le Vif/L'Express) : construction suivant une ligne temporelle (passé-présent-futur).

6 Exemples : l'approche par secteur et l'approche par pays dans l'article «Une stratégie payante » (Le Vif/L'Express ); le Nasdaq-les valeurs technologiques et le 
deux partie de la presse non spécialisée. Les relations d'opposition sont également présentes dans les autres articles, mais de manière secondaire. Elles ne constituent pas l'axe autour duquel se structure le récit (3/8 articles pour la presse non spécialisée, 4/8 pour la presse spécialisée). Ces oppositions mettent en relation des lieux. Nous les envisageons dès lors comme des comparaisons de ces lieux plutôt que comme des mises en concurrence d'éléments. Cette étape de notre étude révèle que les articles étudiés ne sont pas majoritairement construits autour d'une seule relation binaire. Les relations entre les acteurs des textes boursiers doivent être davantage envisagées sous forme de réseau.

Les relations entre les acteurs peuvent aussi être mises en évidence à partir des rôles qu'ils jouent dans le récit en tant qu'actants au sens « greimassien ». L'étude du schéma actantiel pour chaque article nous a montré que l'axe de la communication (destinateur-destinataire) était très faiblement présent. Le récit est en effet essentiellement construit autour de deux axes, celui du désir (sujet-objet) et celui du pouvoir (adjuvant-opposant). Notons, à propos de l'axe du désir, que l'objet « réel » de la quête du sujet boursier n'est jamais explicité. Les articles parlent de « gagner en Bourse », ou d' « augmenter de valeur », mais jamais il n'est véritablement question d'argent. Le schéma actantiel nous donne l'image de la Bourse vue comme un système, une "grande machine » qui fonctionne par et pour elle-même, où le but n'est pas véritablement de gagner de l'argent mais de trouver des stratégies, de découvrir des moments propices pour acheter ou pour vendre, ou pour s'introduire sur le marché. Le rôle des opposants et des adjuvants, situés sur l'axe du pouvoir, prend ici tout son sens puisqu'il s'agit de ne pas tomber dans les pièges boursiers, d'éviter les mauvais conseils, de se méfier de certains agents perturbateurs. Il faut en outre trouver des astuces, saisir sa chance, reconnaître les opportunités et les bons conseils.

A côté de l'étude des acteurs, il nous a semblé intéressant de mettre au jour la structure du récit dans chaque article, en repérant les différents niveaux de construction narrative. Nous avons classé les structures globales des récits en quatre niveaux, du plus haut degré de narrativité au plus faible.

La première construction narrative est celle qui comprend une situation initiale, un élément déclencheur, une série de transformations et une situation finale. Un article, non spécialisé, entre dans cette caté-

Down Jones-les valeurs traditionnelles dans l'article « Le Nasdaq contre le Down

Jones » (Le Vif/L'Express). 
gorie $^{1}$. Le texte commence par mettre en place le contexte et l'axe du désir en présentant le sujet et l'objet de la quête. "Les investisseurs en actions ont longtemps privilégié l'approche par pays et par devise». Interviennent alors deux éléments qui vont « changer la donne » : l'intégration européenne et la mondialisation croissante de l'économie. La diversification par secteur d'activités commence à prendre une place croissante dans les stratégies d'investissements. Pour remplir sa mission, le héros devra franchir deux étapes: trouver les secteurs les plus porteurs en fonction du cycle économique mondial, puis se concentrer sur les actions phares des secteurs concernés. L'investisseur verra à terme les secteurs devenir le critère principal de sélection.

Le deuxième niveau concerne les récits non clôturés et ceux qui effectuent un retour à la situation initiale ou au moment déclencheur, c'est-à-dire les récits en boucle. Quatre articles non spécialisés et un article spécialisé se situent à ce niveau. On retrouve un exemple de récit non clôturés dans l'article «Brokers on line en panne $»^{2}$ où l'auteur se sert d'un événement particulier (le salon du 3ème millénaire) comme déclencheur de son article. Cet événement est un prétexte de l'auteur pour se poser une série de questionnements à la suite l'un de l'autre (Existe-t-il une bourse du 3ème millénaire? Doit-on informer les consommateurs de l'existence de sites transactionnels ? Pour quelles raisons doit-on oser des investissements on line ?) sans y apporter de réelle conclusion. Evoquons à présent un exemple de récit en boucle 3 . Dans l'article «Intéressants les cliquets ? », pour commencer, l'investisseur achète des sicavs à cliquets. Un problème se pose : il ne sait pas se procurer des informations claires et précises à leur sujet et il risque de tomber dans un piège. L'élément déclencheur est donc le manque d'information. Pour déjouer les pièges, l'investisseur doit d'abord lire le prospectus d'émission. Deuxième étape : il doit conserver les sicavs à cliquets jusqu'à l'échéance. Enfin, une fois celles-ci arrivées à terme, il ne doit tenir compte que du rendement réel de son placement. Mais, en définitive, comme les risques ne sont de toute façon pas nuls, il faut dès le départ, dès la situation initiale, ne faire confiance qu'à des institutions qui ont pignon sur rue.

Le troisième type de récit est constitué d'une situation initiale qui correspond à une introduction d'une série de «mini-récits » qui

\footnotetext{
Exemple dans l'article «Une stratégie payante » (Le Vif/L'Express).

Exemple dans l'article «Brokers on line en panne » (La Libre Entreprise).

Exemple dans l'article «Intéressants les cliquets?» (Le Vif/L'Express).
} 
se suivent dans le texte et qui peuvent être construits ou non à partir une structure similaire ${ }^{1}$. C'est le cas pour deux textes non spécialisés et quatre spécialisés. Dans l'article «Manque de visibilité pénalisée » de La Libre Belgique, le constat que « les analystes font preuve d'une grande circonspection face à la discrétion de quelques acteurs de marché » met en place la situation initiale. Se suivent ensuite une série de huit «mini-récits » qui mettent en scène une action et les éléments qui ont guidé les variations de son cours. L'article « Petit parfum spéculatif en ce début d'été » se présente également comme une brochette de mini-récits. L'auteur part d'une situation générale (la détente de l'argent outre-atlantique de la FED) pour en expliquer les conséquences dans divers lieux sous forme de mini-récits clôturés (la situation de Colruyt, de Glaverbel, de KBC, etc).

Le dernier niveau de narrativité est celui où l'article est une pure description, où aucun enchaînement d'actions ou de situations n'est repérable globalement ${ }^{2}$. Un article non spécialisé et trois textes spécialisés sont concernés. Il s'agit par exemple de l'article «La présence d'Interbrew chamboule les statistiques d'Euronext Bruxelles » qui correspond à une simple présentation des chiffres du groupe Interbrew pour l'année 2000 et de sa formule juridique. On ne retrouve aucune « action » dans ce récit, qui n'est est donc peut-être pas un...

Cette dernière analyse révèle que les articles boursiers étudiés, en général, ne respectent pas une structure narrative classique. Les textes de presse spécialisée sont cependant, sur le plan de leur construction, un peu moins narratifs que les non spécialisés.

\section{Quels enjeux pour les représentations et la compréhension?}

Nous avions émis l'hypothèse, a priori, que les textes sur la Bourse ne sont pas construits sur un mode narratif. Or, comme nous avons pu l'observer, la narrativité est présente, même à un faible niveau, et elle joue un rôle au moment de la réception de ces récits par les lecteurs.

De manière générale, on constate un éclatement du récit journalistique dans les articles boursiers, que ce soit dans la presse spécialisée ou

1 Exemples dans les articles : «Manque de visibilité pénalisé » (La Libre Belgique) et «Petit parfum spéculatif en ce début d'été » (La Libre E3ntreprise).

2 Exemple dans l'article «La présence d'Interbrew chamboule les statistiques d'Euronext Bruxelles » (L'Echo). 
non spécialisée. Cette dispersion est illustrée par plusieurs des éléments de narrativité que nous avons évoqués, notamment la désorganisation temporelle et le faible niveau de structure narrative classique comprenant une situation de départ, une transformation et une clôture du récit. Cet éclatement contribue, à notre avis, à véhiculer chez les non initiés une représentation « obscure » de la Bourse. Cette image est complétée, à travers le relevé des schémas actantiels, par celle d'une Bourse qui fonctionne par et pour elle-même, comme une grande machination.

Le fait que le récit sur la Bourse mette toujours en scène plusieurs acteurs centraux en même temps, pouvant même aller jusqu'à cinq dans un même récit, renforce cette idée de dispersion. Le nombre important d'acteurs ne favorise pas non plus l'adhésion du lecteur aux personnages. Les acteurs, bien que personnalisés d'une certaine façon par l'utilisation de champs sémantiques propres, ne sont pas construits et définis dans chaque article comme des personnages véhicules d'affect. Ils ne sont pas ou très peu décrits en terme émotionnel. Ils n'ont pas de personnalité bien spécifique. Nous pensons dès lors que les acteurs, considérés dans chaque article en-dehors du récit médiatique global de la Bourse, ne peuvent pas remplir leur rôle de lieu d'investissement ${ }^{1}$, de facteur d'identification. Or, par ce « pouvoir » d'impliquer le lecteur, le personnage pourrait être une « porte d'entrée » du lecteur dans le texte ${ }^{2}$. Est-ce que nous entendons par là que la construction de personnages au sens narratologique favoriserait la compréhension de la vie économique et boursière ? Est-ce qu'un plus haut degré de narrativité permettrait aux lecteurs de mieux saisir le système boursier? Le personnage, comme lieu d'investissement, produit de la centration, amène le lecteur à se centrer sur tel ou tel acteur. Cette centration constitue un moment du processus de compréhension, mais elle n'en est aussi qu'un temps. Le lecteur ne parvient pas à atteindre un niveau de compréhension global s'il ne développe pas également un processus de décentration, une moment de recul, ce que ne permet peut-être pas l'investissement émotionnel dans un personnage.

Les éléments qui mettent en œuvre la narrativité, comme les personnages, pourraient favoriser l'introduction du lecteur dans le récit boursier mais le récit serait sans doute davantage au service de la compré-

1 Cfr REUTER Yves, «Le personnage », in La question du personnage, Cahiers de Recherche en Didactique du français, n` ${ }^{\circ}$, Clermont-Ferrand, octobre 1987, p. 16 et suiv.

2 ROBINE Nicole citée par REUTER Yves, «Le personnage », op.cit., p. 34 
hension s'il se situait à un niveau plus général de l'histoire économicoboursière, la narrativité ramenant souvent le récit à un certain niveau de « concrétude » et d'exemplification. Quels pourraient dès lors être les schémas narratifs globaux qui aideraient à la compréhension de la vie économique et boursière ? 
